\title{
Optoelectronic Properties of InAIN/GaN Distributed Bragg Reflector Heterostructure Examined by Valence Electron Energy Loss Spectroscopy
}

\author{
A. Eljarrat, ${ }^{1, \star}$ S. Estradé, ${ }^{1,4}$ Ž. Gačević, ${ }^{2}$ S. Fernández-Garrido, ${ }^{2,5}$ E. Calleja, ${ }^{2}$ C. Magén, ${ }^{3}$ \\ and F. Peiró ${ }^{1}$ \\ ${ }^{1}$ Laboratory of Electron NanoScopies, LENS-MIND-IN2UB, Dept. Electrónica, Universitat de Barcelona, Marti i Franqués 1, \\ Barcelona, Spain \\ ${ }^{2}$ ISOM, Instituto de Sistemas Optoelectrónicos y Microtecnología, Universidad Politécnica de Madrid, Spain \\ ${ }^{3}$ Instituto de Nanociencia de Aragón (INA) and Departamento de Física de la Materia Condensada, \\ Universidad de Zaragoza, 50018 Zaragoza, Spain. Fundación ARAID, 50018 Zaragoza, Spain \\ ${ }^{4}$ TEM-MAT, (CCiT), Universitat de Barcelona, Solís i Sabarís 1, Barcelona, Spain \\ ${ }^{5}$ Paul-Drude-Institute for Solid State Electronics, Hausvogteiplatz, 5-10117 Berlin, Germany
}

\begin{abstract}
High-resolution monochromated electron energy loss spectroscopy (EELS) at subnanometric spatial resolution and $<200 \mathrm{meV}$ energy resolution has been used to assess the valence band properties of a distributed Bragg reflector multilayer heterostructure composed of InAlN lattice matched to GaN. This work thoroughly presents the collection of methods and computational tools put together for this task. Among these are zero-loss-peak subtraction and nonlinear fitting tools, and theoretical modeling of the electron scattering distribution. EELS analysis allows retrieval of a great amount of information: indium concentration in the InAlN layers is monitored through the local plasmon energy position and calculated using a bowing parameter version of Vegard Law. Also a dielectric characterization of the InAlN and GaN layers has been performed through Kramers-Kronig analysis of the Valence-EELS data, allowing band gap energy to be measured and an insight on the polytypism of the GaN layers.
\end{abstract}

Key words: VEELS, optical properties, III-V nitrides, Kramers-Kronig analysis, band gap

\section{INTRODUCTION}

Electron probes in aberration-corrected scanning transmission electron microscopy (STEM) can be as small as a few angstroms on the sample and can be successfully combined with monochromated electron energy loss spectroscopy (EELS) that can achieve an energy resolution down to $\sim 0.1 \mathrm{eV}$ (Terauchi et al., 1991; Batson, 2005; Walther et al., 2006; Erni et al., 2008). In general, EELS assesses the energy loss of inelastically scattered electrons that pass through the sample in the transmission electron microscope (TEM). Valence-EELS [(V)EELS] centers itself on the lower energy loss (low-loss) spectrum, typically $<50 \mathrm{eV}$. The features in the low-loss region of the spectrum are identified as incident electron beam interactions with the outer shell (i.e., with valence and conduction band) electrons, thus retrieving valuable information on the local electronic structure of the examined material (the theory was developed by Bethe, 1930 and was generalized in Kröger, 1968; see Egerton, 2009 for an up-to-date account of the application in EELS).

In the present work, we report an extensive (V)EELS characterization of InAlN/GaN distributed Bragg reflector (DBR) structures grown by plasma-assisted molecular beam epitaxy (MBE). By exploiting the low loss region of the EELS spectrum, we assess information regarding determination of indium concentration through the InAlN layers, the

Received January 16, 2012; accepted May 3, 2012.

${ }^{\star}$ Corresponding author. E-mail: aeljarrat@el.ub.edu measure of the band gap energy, and the dielectric characterization of the group III nitride sample material. In the following paragraphs we summarize the main steps to accomplish the task.

Improvements concerning treatment of the spectrum signal for the EELS low-loss region analysis constitute an open area of research. Some information in EELS can be examined almost directly by simply looking at the spectrum, which, in fact, reflects the joint density of states [JDOS, convolution of the valence- and conduction-band densities (Rafferty \& Brown, 1998)], but deeper analysis is needed to obtain quantitative results. Analysis consists in the separation of the theoretically modeled single scattering distribution (SSD) signal, from a variety of other contributions. This signal is generated exclusively by electrons inelastically scattered just once. If the signal is correctly recorded, features in the EELS spectrum, such as band gap energies, plasmon energies, and band transition-related peaks, can be assessed and further examined. The large tails of the zeroloss peak (ZLP) tend to mask the low-energy features, and we have chosen a deconvolution routine to cope with this.

Among the latter spectral characteristics, the plasmon peak is probably the most intense one. The resonance energy of the plasmon vibration, depending on the lattice constant, can be related to the composition of the material through Vegard's law (Erni \& Browning, 2006; Arbiol et al., 2009; Palisaitis et al., 2011). We present a method to accurately measure the energy positions of the plasmon peaks in 
spectra with Lorentzian peak fitting. With the use of Vegard's law plus a bowing parameter to account for nonlinearities to first order, this methodology allows us to monitor the local segregation of a single species in the ternary material InAlN.

By further analysis, the complex dielectric function (CDF), which gives valuable information on the material electronic structure, can be calculated. Bulk energy loss function is related to the SSD by the Kröger formula, and it can be used to obtain the CDF through Kramers-Kronig analysis (KKA, see Egerton, 1996). This procedure normalizes the spectrum using the KK sum relation and a refractive index constant, dealing also with surface-loss signal inside the spectrum. The resulting energy-loss function is cleaner than the SSD, in the sense that ideally the contributions from bulk inelastic losses are separated. The EEL CDF allows access to valuable information on optical properties of the sample and the confirmation of crystalline structure characteristics by investigation of known fingerprints of phase dependent transitions in the dielectric data (Lazar et al., 2003).

The use of a formal treatment is also necessary to understand the effect of delocalization of the inelastic scattering in crystals (Erni \& Browning, 2005). To deal with signal-to-noise ratio (SNR) problems, it is common to either take a series of measurements at the same point and add them, or to measure ZLP and low-loss spectra separately and then apply Fourier-ratio deconvolution with a modeled ZLP (Brockt \& Lakner, 2000; Rafferty et al., 2000; Lazar et al., 2003; Stöger-Pollach, 2008). Open areas of concern are the surface-loss and retardation spurious signals (due to relativistic Cerenkov effect), which lead to recent revision of the investigations of Kröger (1968), and the use of a more complete formula for the problem of inelastic scattering of electrons by thin films (Erni \& Browning, 2008; Stöger-Pollach, 2008; Egerton, 2009; Potapov et al., 2009).

\section{Materials and Methods}

\section{InAlN/GaN Distributed Bragg Reflectors}

Since the early 1990s, much research effort has been focused on group III nitrides. Their direct band gap combined with high excitonic binding energy provides high light emission efficiency. The huge variation of their room-temperature band gap energies, from the infrared $(\mathrm{InN}, 0.69 \mathrm{eV})$ to the ultraviolet (AlN, $6.2 \mathrm{eV}$ ) range, offers a unique possibility to cover the entire visible range with only one group of materials. The development of InGaN active layers for III-nitride optoelectronic devices allowed the fabrication of blue/green/ white light emitting diodes (LED) that revolutionized the LED market (Nakamura \& Fasol, 1997). Fabrication of short-wavelength laser diodes enabled a dramatic increase of optical disc storage capacity (Ikeda \& Uchida, 2002). However, the huge potential offered by this new group of materials has been significantly constrained by several inherent material system concerns. On the one hand, the high in-plane lattice mismatch between the three binaries $(\mathrm{InN}$, $\mathrm{GaN}$, and $\mathrm{AlN}$ ) inevitably leads to defect formation at the heterostructure interfaces, which affects electrical and/or optical properties of the devices. At this stage, the InAlN ternary compound arises as a potential solution for lattice mismatch related problems. With approximately $18 \%$ of indium incorporated, this compound gets in-plane latticematched (LM) to GaN, allowing the growth of strain-free InAlN/GaN heterostructures.

On the second hand, due to the high miscibility gap between InN and AlN binaries, early theoretical calculations based on a strictly regular solution model predicts high mixing instability and strong spinodal decomposition of InAlN material (Matsuoka, 1997). This leads to formation of layers with two separated zones, rich and poor, in In content and/or the appearance of In rich isles and honeycomb structures (Kuzmik et al., 2002; Vilalta-Clemente et al., 2010).

Despite these difficulties, experimental results reported during the previous decade, obtained mainly by metalorganic vapor phase epitaxy (MOVPE), have proven that LM InAlN/GaN heterostructures of excellent quality can be synthesized; high reflectivity DBRs (Carlin \& Ilegems, 2003), resonant-cavity LEDs (Dorsaz et al., 2004), planar microcavities (see Feltin et al., 2005), and optically pumped vertical cavity surface emitting lasers (see Feltin et al., 2007) have already been demonstrated. Reports concerning MBE growth are very scarce and have been focused mainly on high electron mobility transistor structures [in various publications (Katzer et al., 2005; Jeganathan et al., 2007; Dasgupta et al., 2011)]. First systematic studies of the material growth have been reported recently (Ive et al., 2008; FernándezGarrido et al., 2008), as well as short LM InAlN/GaN superlattices and ten-period DBRs (Schmult et al., 2007; Gačević et al., 2009, 2010). Bearing in mind that the MBE technique facilitates formation of flat and abrupt interfaces, it can be noted that in the field of InAlN/GaN heterostructures, the potential of this growth technique has not yet been exploited. The background reason is, above all, the huge difference in optimal growth temperatures for the two binaries $\left(400^{\circ} \mathrm{C}\right.$ and $800^{\circ} \mathrm{C}$ for $\mathrm{InN}$ and $\mathrm{AlN}$, respectively).

In addition to proneness to spinodal decomposition, early theoretical calculations also predicted that as a consequence of the large electronegativity difference between aluminum and indium atoms, a strong deviation from the usual parabolic dependence of InAlN band gap energy on composition can be expected (Dridi et al., 2003). Measurements performed by Iliopoulos et al. (2007) confirmed a strong increase in InAlN bowing parameter with decreasing InN molar fraction, in qualitative agreement with the theoretical findings. This departure from a linear behavior is also detected in our samples, the preparation and examination of which will be accounted for in the following section. It has been recently reported that for AlGaN, another relevant group III tertiary system, both band-gap and plasmon energies followed the chemical composition linearly (Amari et al., 2011). 
In summary, many issues concerning the growth and the crystalline quality of the material remain unclear. It is thus of fundamental importance to determine the structural, optical and electrical properties of these layers, grown by any epitaxial technique, and to correlate them to one another. For this purpose, techniques such as atomic force microscopy (see Kariya et al., 1999), optical reflection spectroscopy, and TEM (see Gačević et al., 2010) have been widely considered. Optical techniques traditionally used for band-gap determination have excellent energy resolution (down to the meV range) but are limited to macroscopic measurements due to their micron-range spatial resolution. However, the very particular nature of the InAlN ternary compound requires characterization techniques providing precise electronic structure information with exceptionally high spatial resolution, as EELS does (Erni \& Browning, 2005, 2006).

\section{Experimental Setup}

Ten-period nearly lattice-matched InAlN/GaN DBRs, with peak reflectivity centered around $400 \mathrm{~nm}$, were grown by a RIBER Compact $21 \mathrm{MBE}$ system equipped with a radiofrequency plasma nitrogen source and standard Knudsen cells for gallium, aluminum, and indium. The InAlN semiperiods were grown under effective stoichiometry at $535^{\circ} \mathrm{C}$ (Ive et al., 2008). This growth temperature provides good InAlN crystalline quality and good control over the targeted $18 \%$ In content. Due to the high thermal sensitivity of In-N bonds, the InAlN semiperiods were buried by consecutive growth of $\mathrm{GaN}$ semiperiods, grown at the same temperature without growth interruptions. As $535^{\circ} \mathrm{C}$ growth temperature is too low for good GaN quality (its optimal growth temperature is $\sim 700^{\circ} \mathrm{C}$ ), excess indium was used as surfactant. Let us note here that a liquid indium layer on the growth surface enhances Ga adatoms surface mobility improving thus the $\mathrm{GaN}$ crystalline quality. Indium atoms incorporate only residually into the growing $\mathrm{GaN}$ crystal (being the InN molar fraction typically below $1 \%$ ). Instead, they accumulate on the growth surface as metal droplets. At the end of each GaN semiperiod, the samples were thermally annealed at temperatures above $600^{\circ} \mathrm{C}$ to allow for desorption of excess indium droplets and to recover a clean and flat surface. To fabricate ten-period DBRs, the same procedure was repeated ten times. More details about sample growth and their structural and optical characterization can be found in our previous publication (Gačević et al., 2010).

The samples were prepared for (S)TEM-EELS observation in cross-section geometry by polishing down to $50 \mu \mathrm{m}$, followed by a dimpling down to $25 \mu \mathrm{m}$ and a final $\mathrm{Ar}^{+}$ bombardment at $V=5 \mathrm{kV}$ and $I=15 \mathrm{nA}$ with an incident angle of $7^{\circ}$ using PIPS-Gatan equipment (Gatan, Inc., Pleasanton, CA, USA). Targeted thickness was $<50 \mathrm{~nm}$ and no significant amorphization occurred, as latter confirmed with high-resolution high-angle annular dark-field (HAADF) experiments. HAADF experiments were carried out in a JEOL J2010F (S)TEM (JEOL Ltd., Tokyo, Japan) and in a probe- corrected FEI Titan (S) TEM (FEI Company, Hillsboro, OR, USA) operated at $300 \mathrm{kV}$ and equipped with a Wien filter monochromator and a Gatan Tridiem 866 ERS energy filter/spectrometer.

Low-loss EEL spectra were acquired by scanning the $\mathrm{GaN}$ and InAlN layers, using the FEI instrument. Spectrum lines were acquired with energy dispersions of 0.01 or 0.02 $\mathrm{eV} /$ channel and with an energy resolution below $0.2 \mathrm{eV}$ as indicated by the ZLP full-width at half-maximum (FWHM). The experimental conditions where $E_{0}=300 \mathrm{keV} ; I=200 \mathrm{pA}$; $\beta, \alpha=17$ mrad; probe size $\sim 0.2 \mathrm{~nm}$.

The limited dynamic range of the EEL spectrometer when collecting the extremely intense ZLP simultaneously with the weakly intense low-loss spectra gives rise to a low SNR of an individual spectrum. While the calculations have been performed individually on each spectrum (unless otherwise stated), mostly spatially averaged spectra are presented as results.

\section{Aim of the Work}

Previous high-resolution electron microscopy (HREM) characterization confirmed expected epitaxial relationships, either between $\mathrm{GaN}$ and sapphire: (0001) [011̄0] $\mathrm{Al}_{2} \mathrm{O}_{3} \|$ (0001) $[11 \overline{2} 0] \mathrm{GaN}$, or between the $\mathrm{GaN}$ and InAlN bilayers: (0001) [112̄0] InAlN \| (0001) [112̄0] GaN (Gačević et al., 2010). Figure 1a shows a general HAADF image of a ten-period DBR. Figure $1 \mathrm{~b}$ displays an atomic resolution image corresponding to one of the GaN/InAlN interfaces observed along the $(11 \overline{2} 0)$ wurtzite-type zone axis. The multilayer grows epitaxially along the (0002) axis of wurtzite structure and presents good lattice matching between the layers. Notice that the GaN layer appears bright at the bottom of the image whereas InAlN appears darker at the top. Between GaN and InAlN, a third region appears with intermediate intensity, which is believed to be an In-rich zone of InAlN, according to the conditions used during the growth of GaN layers with indium acting as a surfactant (Gačević et al., 2010). These high-resolution HAADF images confirm the preparation of a very thin sample $(\sim 50 \mathrm{~nm})$ without amorphization. The objective of the work is then to monitor In segregation in the InAIN layer and to perform a dielectric characterization of the sample. In the following section we will address all the computational methods used for this purpose.

\section{Methods}

All the described procedures and routines have been implemented in Matlab/Octave.

\section{Elastic Signal Removal}

Monochromated STEM-EELS in qualitatively very thin specimens provides exceptional data for low-loss analysis (Arbiol et al., 2009). The most common ways to separate elastic and inelastic contributions to the signal (see the Introduction), and obtain a clean SSD, are based on deconvolution or subtraction methods (Erni \& Browning, 2005; Erni \& 


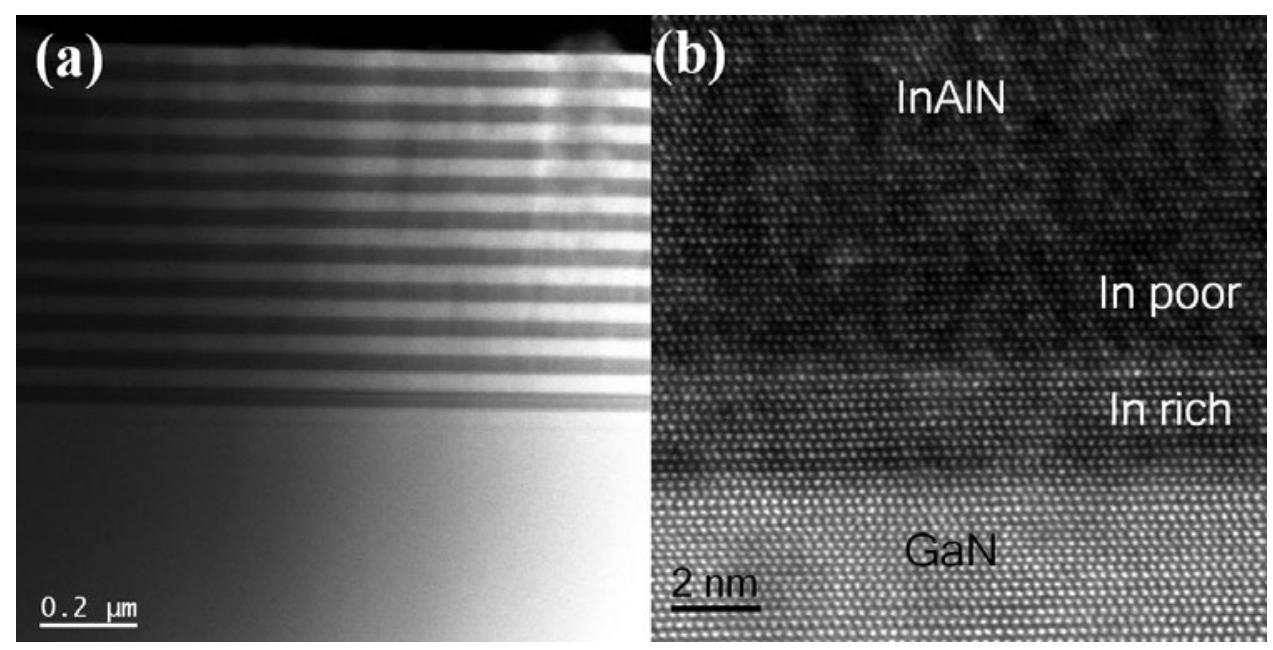

Figure 1. a: STEM HAADF general view of the structure observed along the (1120) wurtzite-type zone axis. b: High-resolution HAADF image of the interface between an InAlN (topmost dark regions) and GaN (bottom layer, lighter), observed along the same direction.

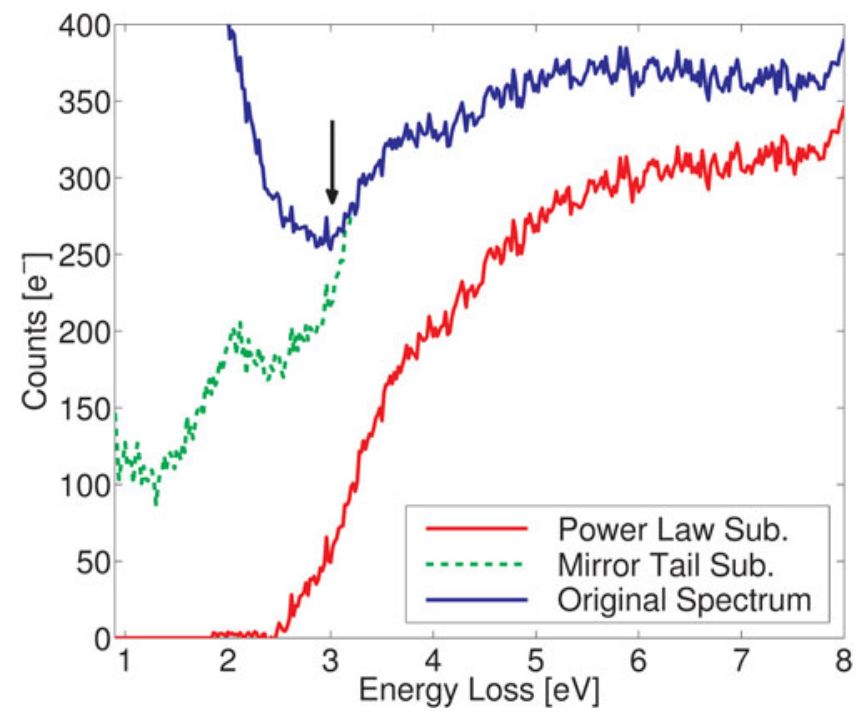

Figure 2. The plot shows the result of the two used tail-subtraction routines together with the original data (20 spectra spatial averaging is performed to increase SNR) from the InAlN layer. The image shows the band gap region in detail. A $1 \mathrm{eV}$ fit interval after the inflexion point was chosen for the power law fit.

Browning, 2007). Subtraction methods have the advantage of being relatively easy to implement, but their results depend on the user ability to find a good tail-fit. The shape and symmetry of the ZLP, as well as the negligible plural scattering contribution (thin sample), allow for the use of simple tail subtraction methods such as power law (PL) fit subtraction or mirrored tail (MT) subtraction.

The first one will fit a PL function to the right end of the ZLP (see Fig. 2). This fit has to be set before the onset of the band gap signal. A computer routine will search for the inflection points in each spectrum, within an energy window, indicating the onset of the inelastic scattering signal. The designed program has a SNR measuring scheme based on the two area method (Egerton, 1996) to help improve the results. The MT method will use the mirrored left part of the ZLP (negative energies) to subtract the elastic contribution only. This method was suggested to be suitable due to symmetric nature of the elastic peak in monochromated experiments (Walther \& Stegmann, 2006; Stöger-Pollach, 2008).

ZLP deconvolution methods were considered in this work, and the chosen method was Fourier-Log, which, as explained in Egerton (1996), is appropriate for low-loss spectra. Implementation of this routine has been carried out with little changes from the original one, adapting the proposed program to fit the energy dispersion of our data. It is important to examine the deconvolved signal and make sure all the desired original features are still present. Especially in the case of very low energies, near the ZLP, when the selection of a good cut-off energy point between ZLP and inelastic signal is crucial to avoid losing valuable information. Again, this cut-off energy was searched separately for each spectrum as an inflexion in the EEL spectrum after the ZLP. Figure 3a shows a raw spectrum from an InAlN region and the result of the deconvolution routine. In the panel below (Fig. 3b), the resulting average of deconvolved spectra from a GaN region is portrayed with some labeled arrows that point to some of the features detected in the registered signal. Below $\sim 5 \mathrm{eV}$, errors are liable to appear as the signal can get smoothed. Yet, this is easily detectable by direct examination, and inelastic SSD with useful information below $\sim 3 \mathrm{eV}$ have been retrieved from the EEL spectra, as presented in the Results and Discussion section.

\section{Indium Ratio Determination}

Once the spectra have been appropriately deconvolved, with an ad-hoc designed routine, we can determine the energy positions of the plasmon peaks along a given spectrum line using a Lorentzian function fit to increase its precision (FWHM $\lesssim 0.2 \mathrm{eV}$ ). The plasmon peak position can be related to the ternary alloy content using a parabolic version 

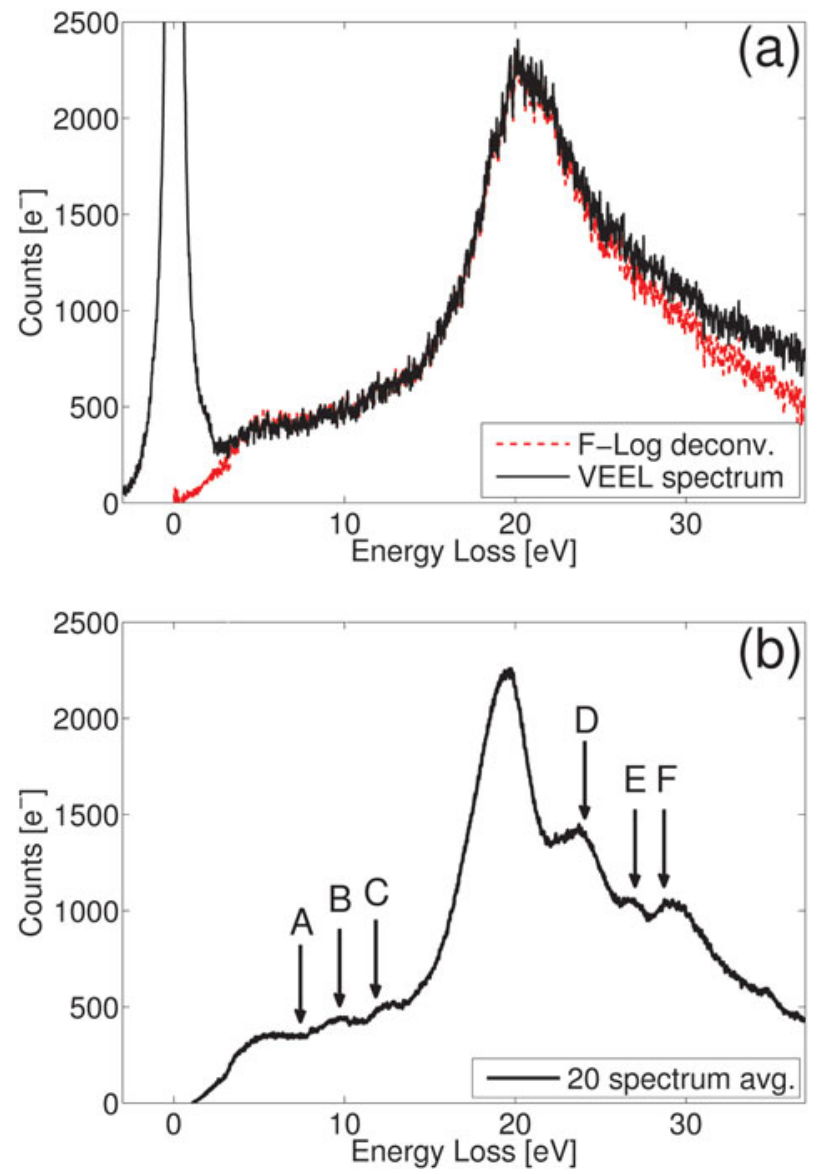

Figure 3. a: Good deconvolution of a single spectrum (no averaging, low SNR) in the InAlN layer; the ZLP is subtracted and information down to the band gap transition signal can be used. b: The result of a 20 spectra average in the GaN layer; letters point to intensity maxima (position is only orientative). The $\mathrm{E}$ arrow points toward a small peak at $\sim 26.7 \mathrm{eV}$.

of Vegard Law. The bowing parameter is derived, imposing the average indium content to $\sim 18 \%$ [the value estimated by X-ray diffraction technique (Gačević et al., 2010)].

This calculation proceeds in the following way: first, the VEEL spectra are aligned using the ZLP position determined through a Gaussian fit to the ZLP, and this peak is then deconvolved using the Fourier-Log deconvolution algorithm. Once the whole spectrum line has been ridden of the ZLP, the next step is to fit an analytic function to the Ep peaks. For this purpose Gaussian and Lorentzian functions were tested; the latter showed better results and were finally chosen (see Fig. 4). These plasmon energies are used to calculate indium concentration, [In]. Recent studies (Palisaitis et al., 2011) have proved that plasmon energy in the $\mathrm{In}_{\delta} \mathrm{Al}_{1-\delta} \mathrm{N}$ system can be related with $\delta$, the In ratio ([In] $=$ $\delta$ ). This $\delta$ will have a variation with the line depth given by Vegard's Law with a bowing parameter, $b$,

$$
E p^{\mathrm{InAIN}}=\delta E p^{\mathrm{InN}}+(1-\delta) E p^{\mathrm{AlN}}-b \delta(1-\delta) .
$$

Mean In content was used for the adjustment of the bowing parameter, as the total In ratio is known to be $\sim 18 \%$. First,

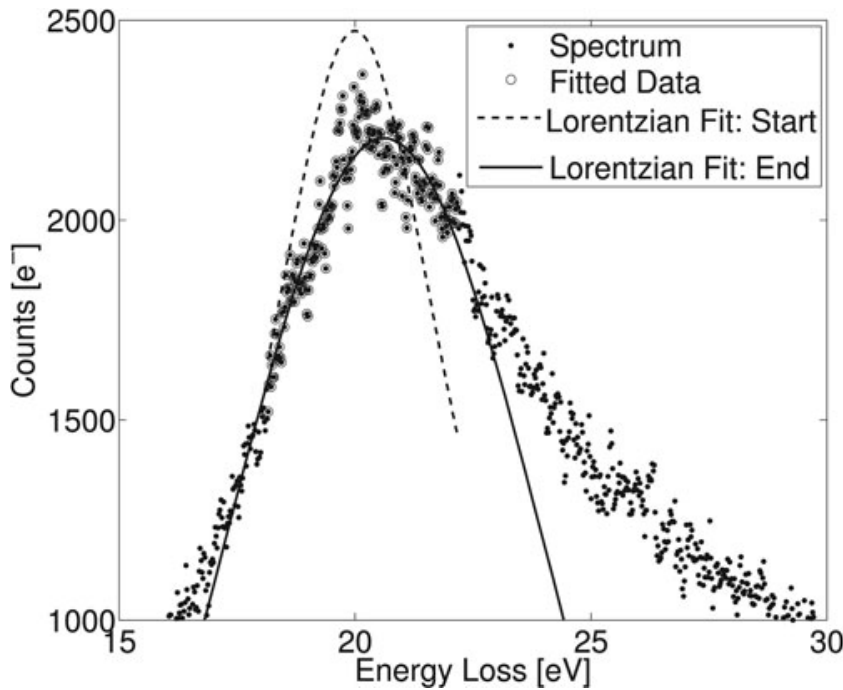

Figure 4. The Lorentzian peak is optimal for plasmon fitting, as predicted by the Drude model. The single spectrum shown, from a InAlN layer, has not been treated by any other method than F-Log deconvolution. The fit uses data points within a symmetric interval $\sim 4 \mathrm{eV}$ wide around the peak maximum.

Vegard's law considering an initial bowing parameter is applied to each spectrum across the InAlN layer [see equation (1)], from which the shape of the graded In concentration profile, $\delta$, is found. The adjustment of the bowing parameter to the indicated nominal value demands the calculation of the integral of all determined indium concentration values. Second, once the average In content can be calculated a bowing parameter is chosen to fit this value to the nominal $\sim 18 \%$. Obtained results are presented in a following section.

\section{Kramers-Kronig Analysis}

Bulk energy-loss function (ELF), $\operatorname{Im}(-1 / \epsilon)$, where $\epsilon$ is the material's $\mathrm{CDF}$, is present in the formula that models the SSD (Kröger, 1968),

$$
\operatorname{SSD}(E)=\frac{I_{\mathrm{ZLP}} \cdot t}{\left[\pi a_{0} 2 T\right]} \cdot \operatorname{Im}\left[-\frac{1}{\epsilon(E)}\right] \cdot \log \left[1+\left(\frac{\beta}{\theta_{E}}\right)^{2}\right] .
$$

This equation is part of a more general expression that accounts for the exiting energy spectrum of an electron beam of kinetic energy $T$ that passes through a thin foil of thickness $t$ with collection semiangle $\beta . \theta_{E}$ is a characteristic inelastic scattering angle for an energy loss $E, I_{\mathrm{ZLP}}$ represents the elastic intensity, and $a_{0}$ is the Bohr atomic radius. This part corresponds only to the contribution of inelastic interactions between the fast electron and the sample electron structure in the dielectric approximation (Egerton, 1996; Kröger, 1968). The inelastic SSD has to be identified and separated from the elastic scattering contribution for all spectra. This is done through Fourier-Log deconvolution as explained in previous sections.

Causality considerations affecting the CDF invite the use of the KK relations to obtain the full CDF from the $\operatorname{Im}(-1 / \epsilon)$. Good use of F-Log deconvolution assures that 
the input signal for KKA contains only the single scattering contribution to the spectra, allowing, thus, for the correct normalization of the ELF. This iterative process, in its simplest case based on normalization through the material refraction index $n_{i}$, has been implemented in our KKA routine, on Matlab, based on the Fortran code krakro.for (Egerton, 1996). It incorporates a calibration section before the iterative loop that calculates the CDF. This calibration consists of the extension of the SSD with a fitted tail of the form $\mathrm{Ar}^{-3}$, as suggested by the Drude model for the plasmon peak. It proves useful in improving sampling for the Fourier-based calculation of $\operatorname{Re}[1 / \epsilon]$. The KKA algorithm not only solves the above formula, it also normalizes the energy-loss function and subtracts the contribution coming from surface losses calculated from the Kröger formula (Kröger, 1968). The resulting CDF of the bulk material is the main objective of the calculations, as it summarizes the electromagnetic information extracted from the spectrum: when the CDFs are finally obtained, at the end of the iterative loop, they are used for the calculation of a number of optoelectronic and structural properties, which will be presented in the Results and Discussion section.

\section{Results And Discussion}

\section{Indium Ratio Determination}

Concerning the possibility of an in-plane composition modulation, the contrast in the HAADF images does not seem to suggest it is taking place (see Fig. 1). In addition, further EELS data have been analyzed to rule out this possibility. High-resolution spectrum images taken parallel to the interfaces were acquired and analyzed, showing no traces of in-plane composition modulation (not shown here).

Indium concentration through the described $\operatorname{In}_{\delta} \mathrm{Al}_{1-\delta} \mathrm{N}$ layers along the growth direction [0001] was estimated via plasmon energy of the locally examined InAlN region (see Fig. 5). The main objective here is to elucidate, using EELS, whether indium is homogeneously incorporated along the growth direction despite the formation of indium droplets during GaN growth. Results are shown in Figure 6. The Ep's for the wurtzite nitride components are taken from the literature (Sánchez-López et al., 1998; Specht et al., 2006), their values are $E p^{\mathrm{GaN}}=19.4 \mathrm{eV} E p^{\mathrm{InN}}=15.7 \mathrm{eV}$, and $E p^{\mathrm{AIN}}=21.1 \mathrm{eV}$. The Ep mean values obtained in the GaN layers are in accordance with the expected plasmon energy of $\mathrm{GaN}$ and measures from other works (Brockt \& Lakner, 2000; Lazar et al., 2003; Arbiol et al., 2009).

According to the lower (red) panel in Figure 6, the In concentration decreases approximately linearly within the first $10 \mathrm{~nm}$ of the InAlN semiperiod from 30\% down to nominal $18 \%$ indium content value. Then, it remains nearly constant at the value slightly below the nominal one until the last few nanometers, where certain increase is appreciated. The existence of two different regions in the InAlN layer, a decreasing-ratio [In] rich region and a poor, and approximately [In] constant region previously noticed in HAADF, is confirmed through (V)EELS. Energy loss preci-

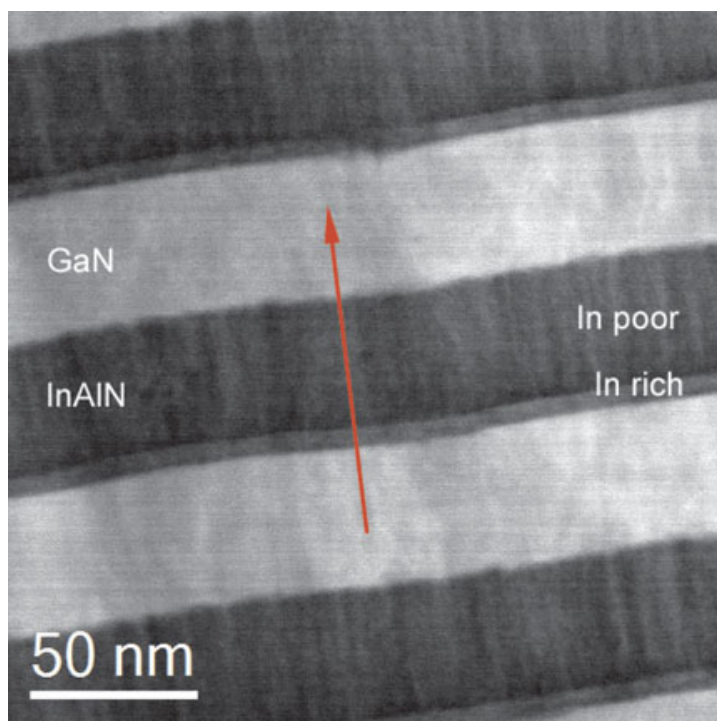

Figure 5. HAADF image showing the examined region. A clear contrast can be appreciated in the InAlN region. The spectrum line has been drawn over the structure, which crosses over all of an InAlN layer, from and to GaN regions.

sion extimated from the ZLPs FWHM dictates an average $\pm 3 \%$ standard deviation on the derived [In] values.

\section{Kramers-Kronig Analysis}

Kramers-Kronig analysis has been performed on the EEL data as explained in the Methods section. Certain calculations in the mathematical routine involve the use of Fourier transformations, as described in Johnson (1975). For fast Fourier transform analysis, it is convenient that all signals start and end at zero (or almost zero) counts, without sharp peaks. At low energies below the cut-off value for deconvolution, negative data points and at least the first channel of all SSD data arrays are set to zero. As the plasmon tail was not measured completely on these spectra because of the chosen $E$ range, the function $\mathrm{Ar}^{-3}$ suggested by the Drude model is used to extrapolate data to zero. Finally, the SSD arrays are padded with zeroes up to twice their actual length, which is bound to reduce the noise coming from sampling in Fourier space.

At this point, refraction index (commonly denoted as $n$ ) values are needed for the normalization loop. The values used for the calculations were taken from the experimental data (Gačević et al., 2010), as were the refractive indices used for normalization of the $\operatorname{ELF}\left(n_{\mathrm{GaN}}=2.5, n_{\mathrm{In}_{0.18} \mathrm{Al}_{0.82} \mathrm{~N}}=2.3\right)$. Different values were tested that did not substantially improve the results, with the exception of using a higher value of $n$ in the In-rich region.

Known refraction indices are necessary for the application of Kramers-Kronig sum rule and normalization of the ELF in KKA. Since the composition of the InAlN layer in the In-rich region is not uniform, the local value of $n$ may vary in this region. Indeed, in the case of the InAlN region, the refraction index values obtained from optical techniques are appropriate for use in our sub-nm-resolved 

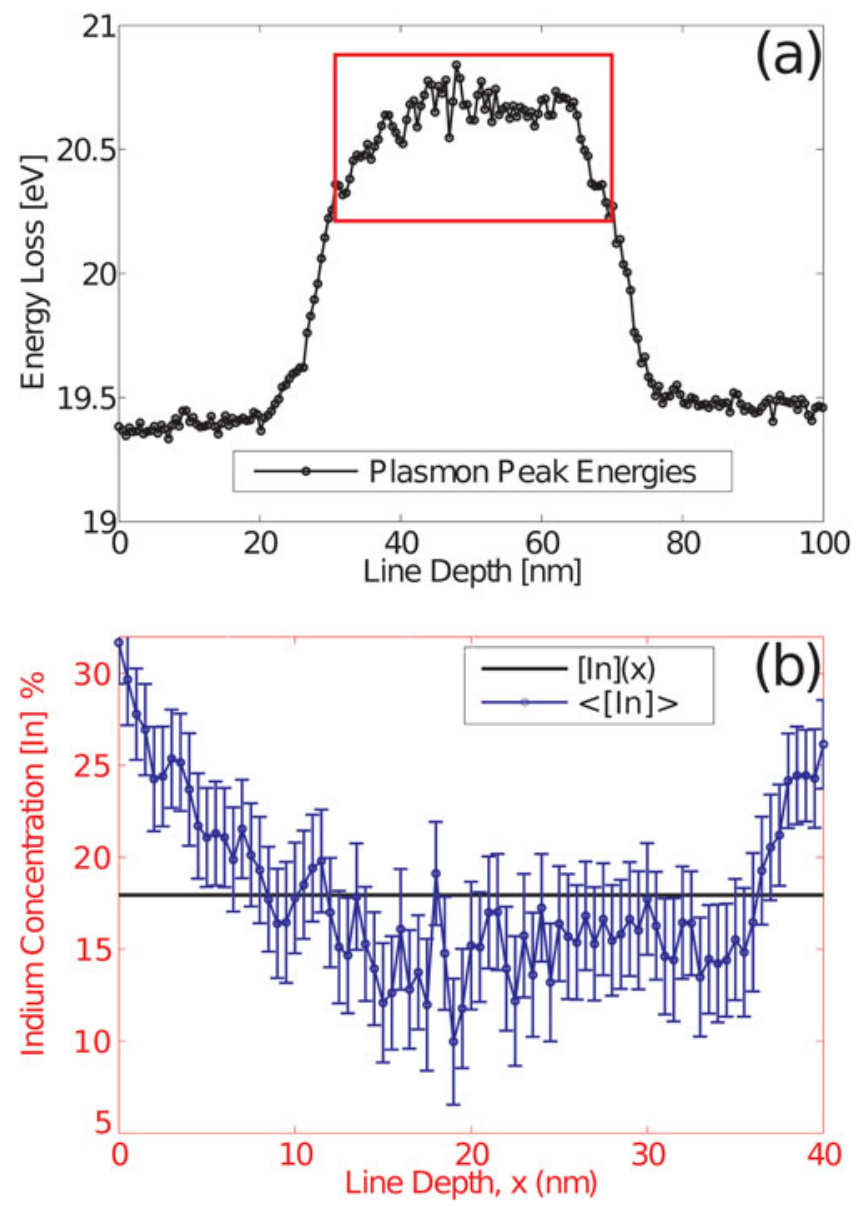

Figure 6. a: The plasmon peak energy positions all through the spectrum line (see Fig. 5), and a red square indicating the InAlN region. b: The In ratio through the $40 \mathrm{~nm}$ thick InAlN layer, as calculated by applying Vegard Law with a bowing parameter fitted to impose the mean $[\mathrm{In}]$ value, $\sim 0.18$, which yields $b \approx-3.1 \mathrm{eV}$. Error bars are included; they were calculated from the plasmon peak determination fits.

spectra. Possible solutions (for the case of this sample) are to check results with the ones of a better known layer, like $\mathrm{GaN}$, or, if possible, to normalize the SSD using a different method, like sample thickness. In our case, it has been preferable to limit the use of the latter $n_{\text {InAlN }}$ to the InAlN layer regions where the In content (plasmon peak position) stays constant, and all the results concerning InAlN exposed in the following will proceed from these data.

Once KKA has been correctly performed, the main result that is obtained is the $\operatorname{CDF}\left(\epsilon=\epsilon_{1}+i \epsilon_{2}\right)$. The CDFs have been recovered point by point, each one corresponding to an individual spectrum. Figure 7 shows an example CDF averaged from adjacent CDFs that were derived from KKA of Fourier-Log deconvolved spectra. This procedure was considered the best possible one to appreciate features coming from lower energy losses. Shapes of the functions are in good agreement with the ones predicted by the Drude model for a semiconductor material (Egerton, 2009): the real and imaginary parts present a crossing point just after the plasmon peak energy and $\epsilon_{1}$ becomes negative indicat-
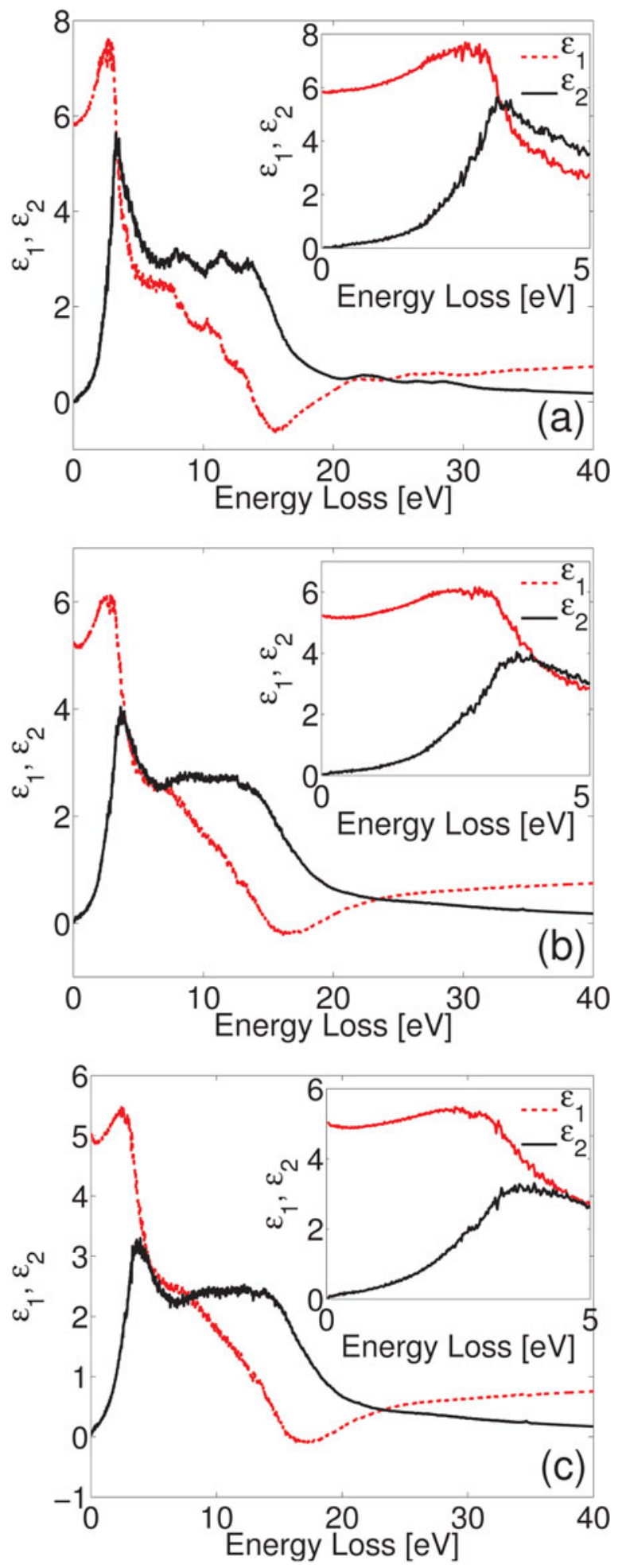

Figure 7. (a) GaN, (b) InAlN In-rich, and (c) InAlN In-poor regions correspond to 20 averaged CDFs from each of the indicated regions. The insets show the low energy region in detail.

ing a transition. Lowest energy values are characterized by $\epsilon_{2}=0$ and $\epsilon_{1} \approx n^{2}$, which is shown in the inset in Figure 7 . Moreover, when $\epsilon_{1}$ decays to negative values after the band gap signal onset, the material will have a dielectric response at energies below the gap value and will behave like a 
conductor at energies above, which is the expected behavior of a semiconducting material.

\section{Optoelectronic and Structural Properties Derived from KKA}

A low band gap signal can be directly observed in the original spectrum, the objective is to retrieve this signal free from the elastic contribution (ZLP). Observed direct band gaps remained close to $\sim 3 \mathrm{eV}$ through the examined $\mathrm{GaN}$ and InAlN layers, as it can be observed in Figure 2 (in the InAlN region) and in the two panels of Figure 3. More precise band gap energy values for selected areas of the examined layers would be highly desirable. They will be calculated from the energy-loss function derived from KKA, through a square root fit.

The normalized ELF that is recovered from KKA corresponds to the JDOS in the conduction and valence bands. Rafferty and Brown (1998) proved that, for a direct band gap transition, a square root function of the shape $A+$ $\left(E-E_{g}\right)^{1 / 2}$ can be used for fitting the ELF band gap peak ( $A$ is a step used to account for background level, see Fig. 8). If one takes advantage of this, it is easy to measure the band gap values once correct and clear ELF have been recovered. After averaging in selected regions of the InAlN and GaN layers to improve the SNR and to obtain sufficiently clear functions, our own Matlab routine fits the square root and plots the band gap transition point value.

The mean result in both cases is almost the same, $E_{g} \approx$ $3.2 \mathrm{eV}$ for $\mathrm{GaN}$ regions, and $E_{g} \approx 3.4 \mathrm{eV}\left[s\left(E_{g}\right) \approx \pm 0.2 \mathrm{eV}\right]$ for most of InAlN (spectra taken from the $[\mathrm{In}] \sim 15 \%$ in the In concentration profile, the almost constant region in Fig. 6b), indicating that the LM InAlN presents a band gap that is close to the one of GaN. In view of these results, it is noticed that the band gap calculation performed after the low-loss data treatment seems to underestimate band gap values for both $\mathrm{GaN}$ and InAlN layers. As far as $\mathrm{GaN}$ is concerned, a band gap of $3.4 \mathrm{eV}$ was expected for pure wurtzite GaN layers. However, previous structural characterization in Gačević et al. (2010) revealed the presence of zinc blende phase as a consequence of using a growth temperature lower than the optimum value for GaN growth, this cubic phase having a theoretical band gap of 3.2-3.3 eV. These two phases appear to be mixed in the GaN semiperiods; the electron beam will cross (and excite) wurtzite and zinc-blende regions anywhere it enters the sampled GaN regions. The coexistence of these hexagonal and cubic phases could give rise to the lower band gap values determined after the (V)EELS analysis. Nevertheless, the inconsistency of the results for the InAlN layer would remain.

The band gap bowing relation between the [In] and the $E_{g}$ of InAlN was recently reviewed in Iliopoulos et al. (2007). This study used spectroscopic ellipsometry on a series of samples grown by MOVPE, covering a wide compositional range, to determine an experimental relation for the InAlN bowing parameter dependence on the alloy composition. According to this study, a band gap value of $E_{g} \sim 4 \mathrm{eV}$ is predicted for an In concentration of $[\mathrm{In}] \sim 18 \%$. In our case, since the In concentration is slightly lower in the considered region, we should expect a band gap value even higher than $4 \mathrm{eV}$, somewhat far from our measurement of $3.4 \mathrm{eV}$. On the other hand, our measured value is in agreement with other experimental results, corresponding to different growth techniques, included in Iliopoulos et al. (2007) and references therein. More recently, other authors have predicted by $a b$ initio simulation an anomalous band gap evolution with composition, and hence lower band gap in good agreement with our results, if In clustering (understanding cluster as a nitrogen environment with a determinated indium coordination number, see Gorczyca et al., 2010) is present in the layer because of the bowing of the valence band level induced by strong interaction between In and neighboring $\mathrm{N}$ atoms. We cannot disregard local In clustering in the InAlN layer, according to the fact that there was an excess of In at the first stages of growth of InAlN layer as previously discussed, although no actual evidence has yet been found.

Despite the remaining controversy about the expected gap values, in particular our measurements could also be affected by relativistic effects, having in mind that our measurements have been performed at $300 \mathrm{kV}$. This point will be addressed in the following subsection.

As a sanity check on KKA data derived, to test the obtained CDFs, Bethe F-Sum Rule routine was designed to calculate the integrals and the results are shown in Figure 9. The integrals are performed through all channels of each of the spectra, and the one that operates with $\epsilon_{2}(E)$ will always have to be a greater value than the other one, which uses the ELF (Egerton, 1996). This is so in our case, which is a good indication. Also, from these integrals an effective number of electrons taking part in the transitions (for unit cell) can be derived, which seems to be $\sim 3$. The high dispersion of values in the middle region in this image, which corresponds to the InAlN layer, reflects the failure in determining a good refractive index for all InAlN regions.

With the obtained CDF, Cole-Cole plots were prepared to help us identify characteristic transitions. The results are shown in Figure 10, where representative plots for GaN and InAlN regions are depicted in Figures 10a and 10b. The one corresponding to InAlN shows no trace of any transition apart from the ribbon shape resulting from the convolved loops of band gap and plasmonic transitions, which are features that we have already examined. On the other hand, the GaN Cole-Cole plot shows additional features that will be commented on below.

According to energy values reported in the literature (Lazar et al., 2003), we can clearly distinguish a characteristic transition at $\sim 7.8 \mathrm{eV}$ corresponding to pure h-GaN as highlighted in both the CDF and Cole-Cole plots in Figures $10 \mathrm{c}$ and $10 \mathrm{~d}$. Additional transitions are also detected at energies $\sim 10,12.2,23.6$, and $28.9 \mathrm{eV}$ that can be attributed to both the h- and c-GaN phases (values from Lazar et al., 2003). Moreover, at high energies more small loops give hints of three transitions corresponding to energy values above the band gap (one of them at $\sim 26.7 \mathrm{eV}$, characteristic of pure $\mathrm{c}-\mathrm{GaN})$. 
Line Depth $\approx 3 \mathrm{~nm}$, Band Gap Energy $\approx 3.24 \mathrm{eV}$

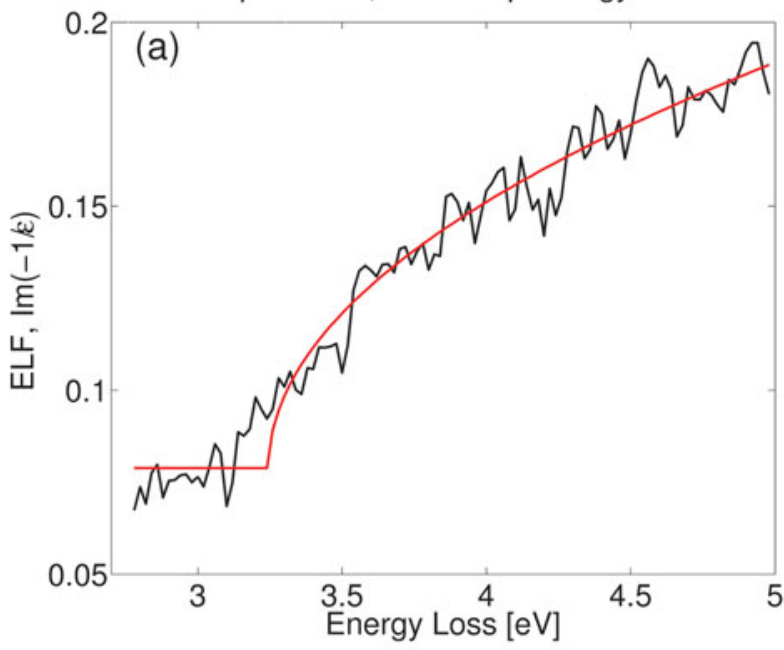

Line Depth $\approx 66 \mathrm{~nm}$, Band Gap Energy $\approx 3.38 \mathrm{eV}$

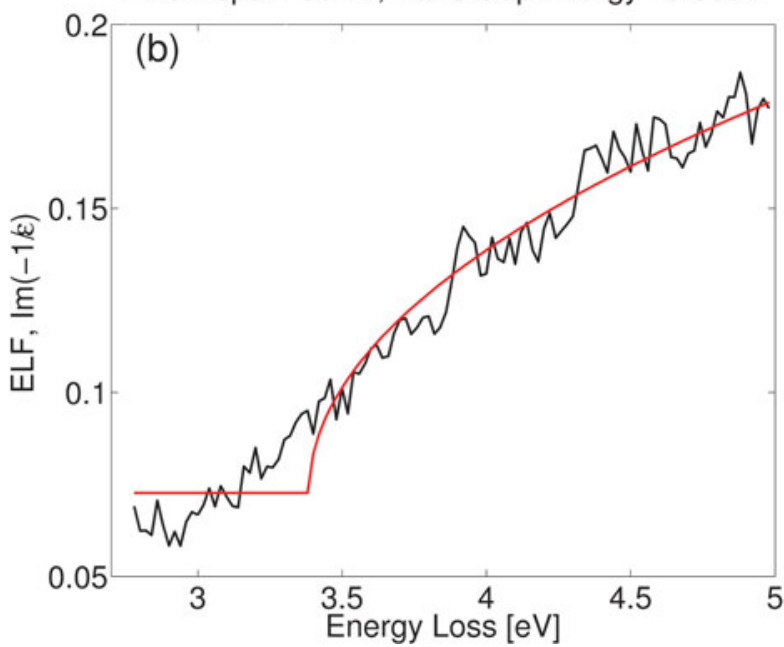

Figure 8. Square root fit, individual examples from $\mathrm{GaN}$ region (a) and InAlN (In-poor region, b). Only the data inside the intervals used for these fits has been plotted (from $\sim 2.8$ to $5 \mathrm{eV}$ ). The added step accounts for the departure of the data from a square root shape at the baseline. The values of the $E_{g}$ are indicated. Plots correspond to a 4 spectra average.

Looking back to low-loss spectra of the very same data (the CDF depicted in Fig. 10c corresponds to the SSD of $\mathrm{GaN}$ in Fig. 3), we can correlate intensity maxima (features A to $\mathrm{F}$ ) with the corresponding transitions in the CDF plot. All of these features in Figure 3, except A and E, can be related to both c-GaN and h-GaN. Although slightly visible in the plot, the A peak of pure h-GaN at $7.8 \mathrm{eV}$ can be identified. Feature E, clearly discernible by naked eye, corresponds to the characteristic transition of $\mathrm{c}-\mathrm{GaN}$ at $\sim 26.7 \mathrm{eV}$.

Summing up all this information, our spectra show characteristic transitions from $\mathrm{h}-\mathrm{GaN}$ and c-GaN. As shown by HREM techniques in Gačević et al. (2010), the examined $\mathrm{GaN}$ layers positively are a mixture of wurtzite $(\mathrm{h}-\mathrm{GaN})$ and zinc-blende (c-GaN) structures, and their contributions would have mixed up in the averaged CDF as in the low-loss spectra. Conversely, InAlN layers are homogeneous hexagonal structures. In this work we have confirmed that (V)EEL

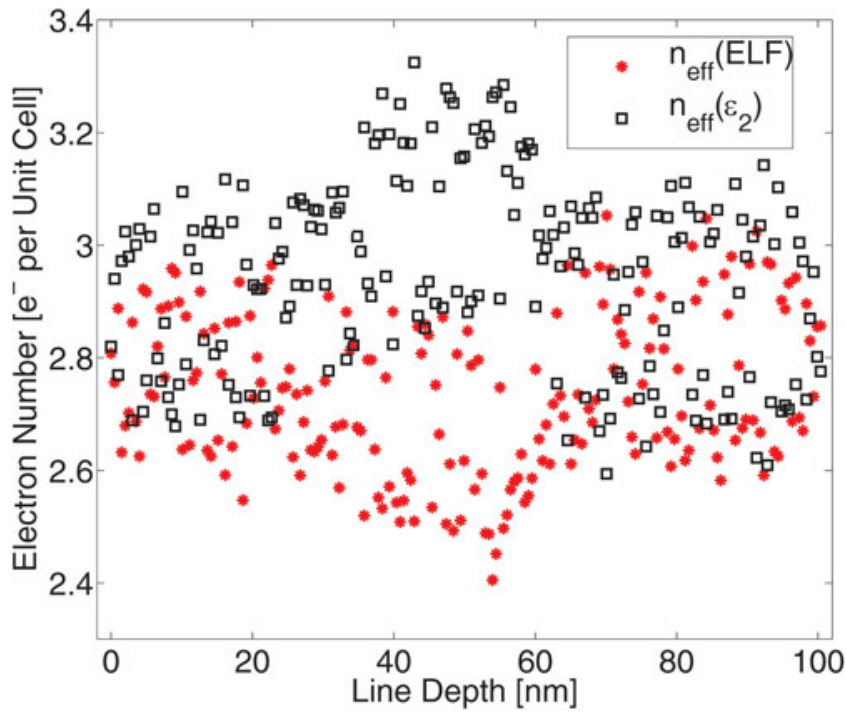

Figure 9. Effective electron numbers resulting from computing Bethe F-Sum rule integrating the ELF (red) or the real part of the $\operatorname{CDF}\left(\epsilon_{2}\right.$, blue) from zero to the end of recorded energy. As expected, blue values are bigger than red ones (Egerton, 1996), which tend to approach them as the integration limit increases.
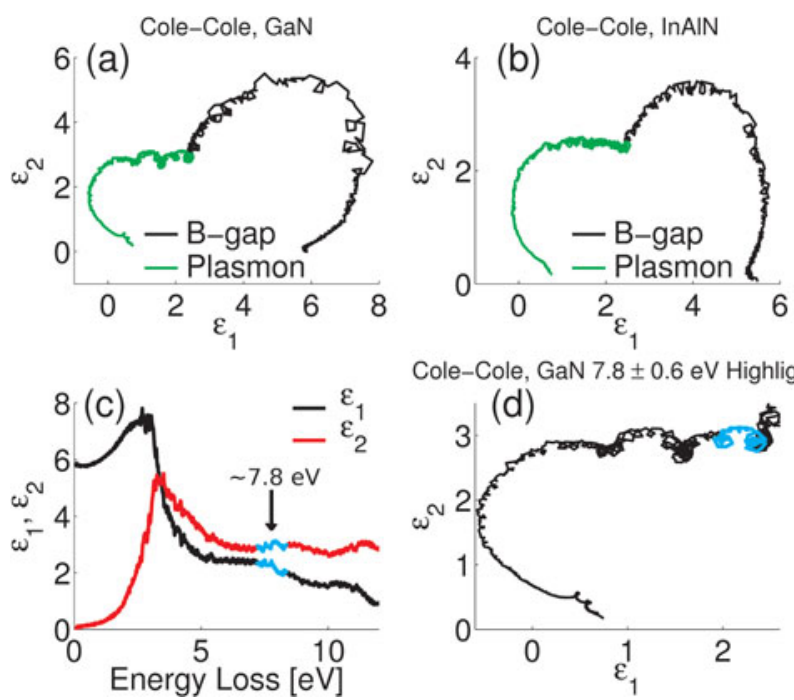

Cole-Cole, GaN 7.8 $\pm 0.6 \mathrm{eV}$ Highlighted

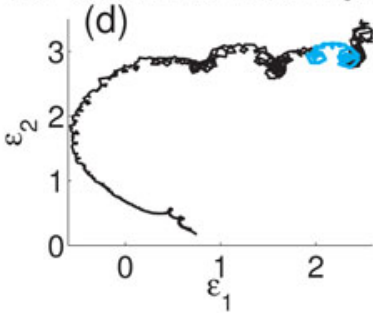

Figure 10. a, b: Examples of GaN and InAlN Cole-Cole plots from the obtained CDFs. Band gap (black, from 0 to $\sim 6 \mathrm{eV}$ ) and plasmon (green or light gray, from $\sim 6$ to $\sim 38 \mathrm{eV}$ ) transition regions are colored to allow easy identification. Subtler transition regions of the CDF can be identified in the panels $\mathbf{c}$ and $\mathbf{d}$. $\mathbf{c}$, $\mathbf{d}$ : Detailed images of a CDF and its corresponding Cole-Cole plots, with a highlighted zone (color version) corresponding to an h-GaN transition $(\sim 7.8 \mathrm{eV}$, A in Fig. 3). All these correspond to 20 averaged CDFs from each of the indicated regions.

spectra can be used to detect the mixture of the two polytypes by the appearance of their distinctive features.

\section{Impact of Retardation Losses}

Retardation effects can significantly alter the shape of the obtained CDF (Stöger-Pollach, 2008; Potapov et al., 2009) because of the importance of these effects in the low-loss 
region, depending on the conditions of the experiment. Similar experimental conditions to the ones in this experiment (see the Experimental Setup section) had been already considered in Erni and Browning (2008), in which the impact of retardation losses on the SSD was taken into account using the complete Kröger formulation (Kröger, 1968). In this spirit, and using the procedure described by Erni and Browning, we calculate the difference between the experimentally obtained SSD and the SSD that correspond to the obtained CDF in the complete Kröger formulation as formulated in Erni and Browning (2008).

A complete calculation of the energy loss distribution of the electrons involves several steps. Starting from the CDF that is obtained from KKA, e.g., the ones obtained in the last subsection, Kröger's equation with all loss terms is solved for each point of an angular grid representing the collection aperture. These terms span from bulk and surface losses in a relativistic formulation including retardation losses. A rather costly calculation integrates in the angular domain, and the resulting data block contains the scattering distribution for that case, which is then normalized to allow comparison with the, already normalized, experimental distribution.

To evaluate the impact of retardation losses, it is useful to use a plot with both experimental and theoretical SSD and their difference. The results of this procedure are plotted in Figure 11, showing the predicted analytical shape of the scattering, which is not accounted for in Figure 2. These affect most of the spectrum as a background signal, which is the registered signal that is present before the onset of the band gap. But, although their maximum is located near the band gap onset, the level of the signal is kept lower than the rest of the spectrum so we can rely on the fitting of the square root with constant background profile giving us a measure of the band gap signal energy onset consistent with our error bars.

\section{CONCLUSIONS}

Optoelectronic properties of InAlN/GaN DBRs have been successfully extracted from a combination of experimental data collected at high resolution (both spatially and in energy) and advanced theoretical and computational methods for the analysis of these data.

Plasmon energy position has been determined for InAlN layer with $\sim 0.2 \mathrm{eV}$ accuracy, and the In ratio in the layers was obtained. For this part we have used a procedure based on Vegard's Law. Two different regions have been identified according to their In concentrations (In rich and In poor) in good agreement with high-resolution HAADF images of the layers.

Dielectric characterization has been carried out, with the CDF of the sample recovered and used for practical purposes, e.g., pointing out the polytypism in the GaN layers. Band gap examination of the InAlN LM to GaN multilayer has been also carried out in this work. Computational tools crucial for this part of the analysis involved the subtraction of the zero-loss signal from the spectrum. The
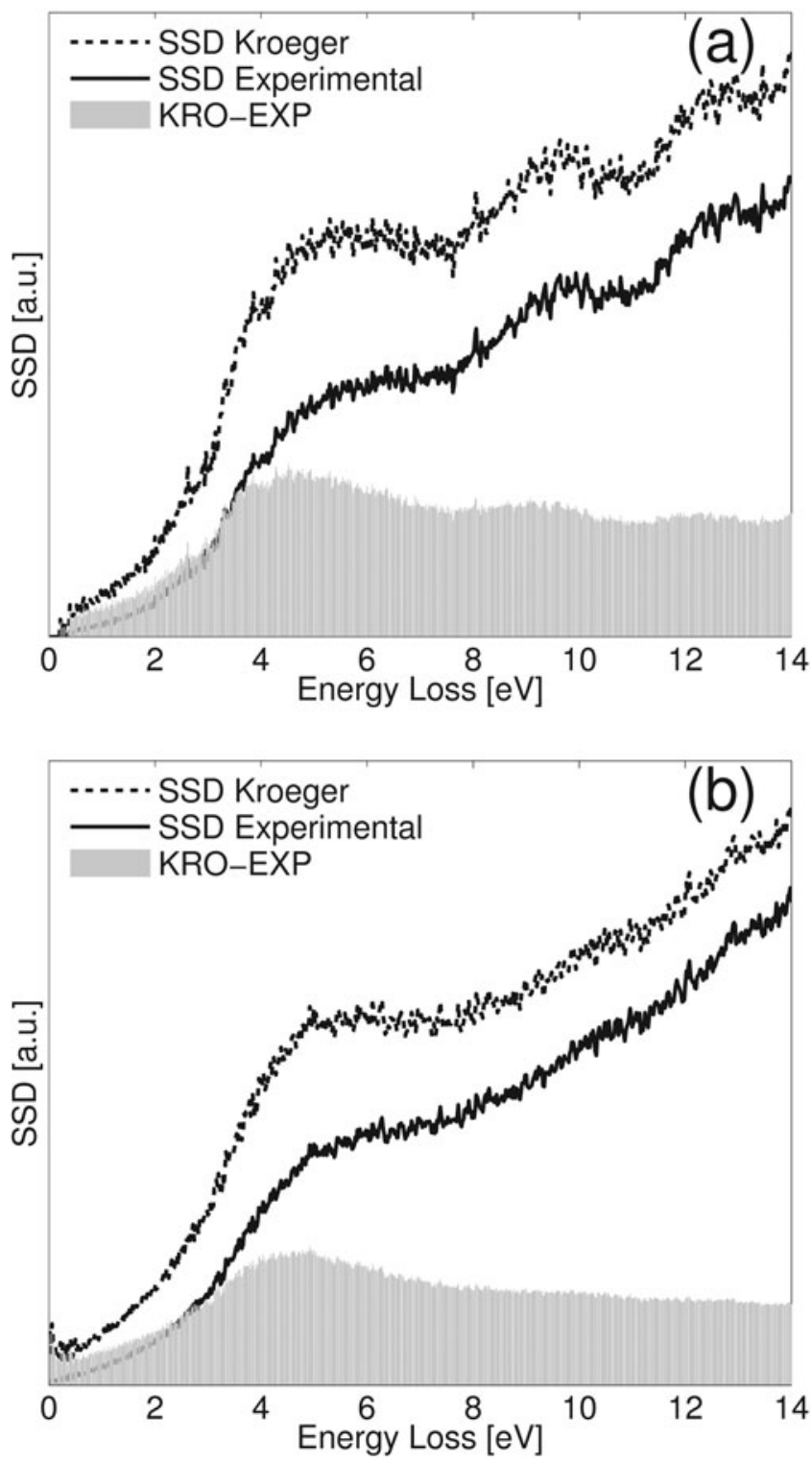

Figure 11. Expected complete scattering for the $\epsilon(E)$ deduced by KKA: comparisons with the experimental SSD and their difference. Panel a corresponds to $\mathrm{GaN}$ region; panel b to InAlN, corresponding to 10 spectra average.

analysis has shown that the energy band gap signal onset remains between 3.2 in the $\mathrm{GaN}$ and $3.4 \mathrm{eV}$ for the InAlN layers, even if with a $\pm 0.2 \mathrm{eV}$ error bar.

The characterization of the electronic contribution to the local dielectric constant of the sample in a subnanometric scale, and the effect of retardation losses has been considered. (V)EELS has allowed us to probe the local optoelectronic properties of InAlN/GaN DBRs.

\section{ACKNOWLEDGMENTS}

We are indebted to Raúl Arenal and M. Stöger-Pollach for the attention and enlightening discussions provided to us, Davood Hosseini who helped with the preparation of the samples, and also Lluís Yedra and F.M. Tarzán-Lorente who 
helped with parts of the code. The Laboratorio de Microscopías Avanzadas (LMA) of the University of Zaragoza and the TEM facilities of the Scientific and Technological Centres (CCiT) of the Univeristy of Barcelona are acknowledged. This work has been carried out within the framework of MAT-2010-16407 and CSD-2009-00013 projects from Spanish Ministerio de Ciencia e Innovación and also CAM (P2009/ESP-1503) project.

\section{REFERENCES}

Amari, H., Zhang, H.Y., GeelhaAr, L., Cheze, C., Kappers, M.J. \& Walther, T. (2011). Nanoscale EELS analysis of elemental distribution and band-gap properties in AlGaN epitaxial layers. In 17th International Conference on Microscopy of Semiconducting Materials 2011, J Phys Conf Series, 326, 012039-012045.

Arbiol, J., Estradé, S., Prades, J., Cirera, A., Furtmayer, F., Stark, C., Laufer, A., Stutzmann, M., Eickhoff, M., Gass, M.H., Bleloch, A.L., Peiró, F. \& Morante, J.R. (2009). Tripletwin domains in $\mathrm{Mg}$ doped GaN wurtzite nanowires: Structural and electronic properties of this zinc-blende-like stacking. Nanotechnology 20, 145704-145713.

Batson, P.E. (2005). Challenges and opportunities of Angstromlevel analysis. In Microscopy of Semiconducting Materials, Springer Proc Phys 107, 451-458.

Bethe, H. (1930). The theory of the passage of rapid neutron radiation through matter. Ann Phys (Leipzig) 5, 325-400.

Brockt, G. \& LAKner, H. (2000). Nanoscale EELS analysis of dielectric function and bandgap properties in $\mathrm{GaN}$ and related materials. Micron 31, 435-440.

Carlin, J.F. \& Ilegems, M. (2003). High-quality AlInN for high index contrast Bragg mirrors lattice matched to GaN. Appl Phys Lett 83, 668-671.

Dasgupta, S., Nidhi, Choi, S., Wu, F., Speck, J.S. \& Mishra, U.K. (2011). Growth, structural, and electrical characterizations of $\mathrm{N}$-polar InAlN by plasma-assisted molecular beam epitaxy. Appl Phys Express 4, 045502-045505.

Dorsaz, J., Carlin, J.F., Zellweger, C.M., Gradecak, S. \& Ilegems, M. (2004). InGaN/GaN resonant-cavity LED including an AlInN/ GaN Bragg mirror. Phys Stat Sol 201, 2675-2678.

Dridi, Z., Bouhafs, B. \& Ruterana, P. (2003). First-principles investigation of lattice constants and bowing parameters in wurtzite AlxGa1-xN, InxGa1-xN and InxAl1-xN alloys. Semicond Sci Tech 18, 850-856.

Egerton, R.F. (1996). EELS in the Electron Microscope. New York: Plenum Press.

Egerton, R.F. (2009). Electron energy-loss spectroscopy in the TEM. Rep Mod Phys 72, 016502-016527.

Erni, R. \& Browning, N.D. (2005). Valence electron energy-loss spectroscopy in monochromated scanning transmission electron microscopy. Ultramicroscopy 104, 176-192.

ERni, R. \& Browning, N.D. (2006). Local indium segregation and bang gap variations in high efficiency green light emitting InGaN/GaN diodes. Sol State Comm 137, 230-234.

Erni, R. \& Browning, N.D. (2007). Quantification of the sizedependent energy gap of individual CdSe quantum dots by valence electron energy-loss spectroscopy. Ultramicroscopy 107, 267-273.

ERni, R. \& Browning, N.D. (2008). Prospects for analyzing the electronic properties in nanoscale systems by VEELS. Ultramicroscopy 108, 84-99.
Erni, R., Lazar, S. \& Browning, N.D. (2008). The impact of surface and retardation losses on valence electron energy-loss spectroscopy. Ultramicroscopy 108, 207-224.

Feltin, E., Butté, R., Carlin, J.F., Dorsaz, J., Grandjean, N. \& Ilegems, M. (2005). Lattice-matched distributed Bragg reflectors for nitride-based vertical cavity surface emitting lasers. Electron Lett 41, 94-95.

Feltin, E., Christmann, G., Dorsaz, J., Castiglia, A., Carlin, J.-F., Butté, R., Grandjean, N., Christopoulos, S., von Högersthal, G.B.H., Grundy, A.J.D., Lagoudakis, P.G. \& BAumberg, J.J. (2007). Blue lasing at room temperature in an optically pumped lattice-matched AlInN/GaN VCSEL structure. Electron Lett 43, 924-926.

Fernández-Garrido, S., Gačević, Ž. \& Calleja, E. (2008). A comprehensive diagram to grow InAlN alloys by plasma-assisted molecular beam epitaxy. Appl Phys Lett 93, 191907-191910.

GaČević, Ž., Fernández-Garrido, S., Calleja, E., Luna, E. \& Trampert, A. (2009). Growth and characterization of latticematched InAlN/GaN Bragg reflectors grown by plasma-assisted molecular beam epitaxy. Phys Stat Sol C 6, S643-5645.

GaČević, Ž., Fernández-Garrido, S., Hosseini, D., Estradé, S., Peiró, F. \& Calleja, E. (2010). InAlN/GaN Bragg reflectors grown by plasma-assisted molecular beam epitaxy. J Appl Phys 108, 113117-113124.

Gorczyca, I., Suski, T., Christensen, N.E. \& Svane, A. (2010). In-clustering induced anomalous behavior of band gap in InAlN and InGaN. Phys Stat Sol C 7, 1283-1286.

IkedA, M. \& UCHIDA, S. (2002). Blue-violet laser diodes suitable for Blu-ray disk. Phys Stat Sol 194, 407-413.

Iliopoulos, E., Adikimenakis, A., Giesen, C., Heuken, M. \& Georgakilas, A. (2007). Energy bandgap bowing of InAlN alloys studied by spectroscopic ellipsometry. Appl Phys Lett 92, 191907-191910.

Ive, T., Brandt, O., Kong, X., Trampert, A. \& Ploog, K.H. (2008). (Al,In) N layers and (Al,In)N/GaN heterostructures grown by plasma-assisted molecular beam epitaxy on $6 \mathrm{H}$ SiC(0001). Phys Rev B 78, 035311-035320.

Jeganathan, K., Shimizu, M., Okumura, H., Yano, Y. \& Akutsu, N. (2007). Lattice-matched InAlN/GaN twodimensional electron gas with high mobility and sheet carrier density by plasma-assisted molecular beam epitaxy. J Cryst Growth 304, 342-345.

Johnson, D.W. (1975). Fourier series method for numerical Kramers-Kronig analysis. J Phys A 8, 490-495.

Kariya, M., Nitta, S., Yamaguchi, S., Amano, H. \& Akasaki, I. (1999). Structural properties of InN on GaN grown by metalorganic vapor-phase epitaxy. J Appl Phys 38, L984-L986.

Katzer, D.S., Storm, D.F., Binari, S.C., Shanabrook, B.V., Torabi, A., Zhou, L. \& Sмith, D.J. (2005). Molecular beam epitaxy of InAlN/GaN heterostructures for high electron mobility transistors. J Vac Sci Tech B 23, 1204-1208.

KRöGER, E. (1968). Calculations of energy losses of fast electrons in thin foils with retardation. $Z$ Phys 216, 115-135.

Kuzmik, J., Javorka, P., Alam, A., Marso, M., Heuken, M. \& Kondos, P. (2002). Annealing of Schottky contacts deposited on dry etched AlGaN/GaN. Semicond Sci Tech 17, L76-L78.

Lazar, S., Botton, G.A., Wu, M.-Y., TichelaAr, F.D. \& ZandberGEN, H.W. (2003). Materials science applications of HREELS in near edge structure analysis and low-energy loss spectroscopy. Ultramicroscopy 96, 535-546.

Matsuoka, T. (1997). Calculation of unstable mixing region in wurtzite In1-x-yGaxAlyN. Appl Phys Lett 71, 105-107. 
Nakamura, S. \& Fasol, G. (1997). The Blue Laser Diode. Berlin: Springer.

Palisaitis, J., Hsiao, C.-L., Junai, M., Xie, M., Darakchieva, V., Carlin, J.F., Birch, N.G.J., Hultman, L. \& Persson, P.O.A. (2011). Standard-free composition measurements of $\mathrm{Al}(\mathrm{x}) \operatorname{In}(1-\mathrm{x}) \mathrm{N}$ by low-loss electron energy loss spectroscopy. Phys Stat Sol RRL 5, 50-52.

Potapov, P.L., Engelmann, H.-J., Zschech, E. \& Stöger-Pollach, M. (2009). Measuring the dielectric constant of materials from valence EELS. Micron 40, 262-268.

Rafferty, B. \& Brown, L.M. (1998). Direct and indirect transitions in the region of the band gap using electron-energy-loss spectroscopy. Phys Rev B 58, 10326-10337.

Rafferty, B., Pennycook, S.J. \& Brown, L.M. (2000). Zero loss peak deconvolution for bandgap EEL spectra. J Electron Microsc 49, 517-524.

Sánchez-López, J.C., Contreras, L., Fernández, A., GonzálezElipe, A.R., Martín, J.M. \& Vacher, B. (1998). AlN thin films prepared by ion beam induced chemical vapour deposition. Thin Solid Films 317, 100-104.

Schmult, S., Siegrist, T., Sergent, S.M., Manfra, M.J. \& Molnar, R.J. (2007). Optimized growth of lattice-matched InxAl1-xN/GaN heterostructures by molecular beam epitaxy. Appl Phys Lett 90, 021922-021925.
Specht, P., Ho, J.C., Xu, X., Armitage, R., Weber, E.R., Erni, E. \& Kisielowski, C. (2006). Zincblende and wurtzite phases in InN epilayers and their respective band transitions. J Cryst Growth 288, 225-229.

Stöger-Pollach, M. (2008). Optical properties and bandgaps from low loss EELS: Pitfalls and solutions. Micron 39, 1092-1110.

Terauchi, M., Kuzuo, R., Satoh, F., Tanaka, M., Tsuno, K. \& Ohyama, J. (1991). Performance of a new high resolution electron energy loss spectroscopy microscope. Microsc Microanal Microstruc 2, 351-355.

Vilalta-Clemente, A., Poisson, M.A., Behmenburg, H., Giesen, C., Heuken, M. \& Ruterana, P. (2010). The structure of InAlN/GaN heterostructures for high electron mobility transistors. Phys Stat Sol 207, 1105-1108.

Walther, T., Quandt, E., Stegmann, H., Thesen, A. \& Benner, G. (2006). First experimental test of a new monochromated and aberration-corrected $200 \mathrm{kV}$ field-emission scanning transmission electron microscope. Ultramicroscopy 106, 963-969.

Walther, T. \& Stegmann, H. (2006). Preliminary results from the first monochromated and aberration corrected $200-\mathrm{kV}$ fieldemission scanning transmission electron microscope. Microsc Microanal 12, 498-505. 